\title{
ON WEAK INTERACTIONS AT HIGH ENERGIES
}

Thomas Appelquist and James D. Bjorken

\section{ERRATA}

We lost a minus-sign, which Carl Albright helped us to find. This propagates through many equations, as noted below, but affects none of our basic conclusions (or less basic conjectures).

p. 11 Eq.(2.16) replace $\frac{9}{4}$ by $\frac{1}{4}$

p. 13 Eq. (3.3) replace the 5 minus signs on the right-hand side by plus signs

p. 14 Replace the first sentence by the following: "The plus signs in Eq. (3.3) occur because $(-1)^{2}=+1$ : one minus sign comes from Fermi statistics and the other from Fierz transformation of the spinors."

p. 14 Eq. (3.6) replace the 5 minus signs on the right-hand side by plus signs. Also

$$
\begin{aligned}
\operatorname{Im} b_{j t}(t) & =2 \operatorname{Re} b_{j t}^{*}(t)[\cdots \\
\operatorname{Im} c_{j t}(t) & =\ldots
\end{aligned}
$$

p. 15 Line 2: "Thus to second order [we assume CP conservation so that all subtraction constants are real]"

p. 15 Eq. (3.10) The second group of equations should be

$$
\begin{aligned}
& (48 \pi)^{2} \operatorname{Im} \mathrm{a}_{1 \mathrm{t}}=2 \alpha_{10}\left(\alpha_{10}+\gamma_{10}\right)(\mathrm{Gt})^{2} \\
& (48 \pi)^{2} \operatorname{Im} \mathrm{b}_{1 \mathrm{t}}=2 \gamma_{10}\left(\gamma_{10}+\alpha_{10}\right)(\mathrm{Gt})^{2}+4 \beta_{10}^{2}(\mathrm{Gt})^{2} \\
& (48 \pi)^{2} \operatorname{Im~} c_{1 \mathrm{t}}=2 \beta_{10}\left(\gamma_{10}+\alpha_{10}\right)(\mathrm{Gt})^{2}+4 \gamma_{10} \beta_{10}(\mathrm{Gt})^{2}
\end{aligned}
$$


p. 15 Line $3 \ldots$ can be constructed ...

p. 16 Eq. (3.11)

$$
\begin{aligned}
& \mathrm{A}(\mathrm{s}, \mathrm{t})=\ldots+\frac{\alpha_{10}\left(\alpha_{10}+\gamma_{10}\right)}{24 \pi^{2}} \text { GsGt } \log (-\mathrm{Gt})+ \\
& \mathrm{B}(\mathrm{s}, \mathrm{t})=\ldots+\frac{\left[\gamma_{10}\left(\gamma_{10}+\alpha_{10}\right)+2 \beta_{10}^{2}\right]}{24 \pi^{2}} \text { GsGt } \log (-\mathrm{Gt})+\ldots \\
& \mathrm{C}(\mathrm{s}, \mathrm{t})=\ldots+\frac{\beta_{10}\left(3 \gamma_{10}+\alpha_{10}\right)}{24 \pi^{2}}+\ldots
\end{aligned}
$$

p. 18

Line $4 \ldots-\frac{1}{\pi}(\mathrm{Gs})^{\mathrm{n}} \log (-\mathrm{Gs}) \ldots$

Line $6 \ldots-\frac{1}{2 \pi}(G s)^{n} \log ^{2}(-G s) \ldots$

p. 20

Eq. (3.23) ${ }^{\circ} \alpha_{1 \mathrm{~m}}=\ldots$

p. 21

Eq. (4.1) $D(s, t)=C(s, t)+A(s, u)$

$\mathrm{Eq} \cdot(4.4) \quad \lim _{\mathrm{s} \rightarrow 0} \frac{\mathrm{D}(\mathrm{s}, 0)}{\mathrm{s}}=\left(\gamma_{10}+\alpha_{10}\right) \mathrm{G}=\ldots$

p. 23 Line $14 \ldots$ behavior of ...

p. $24 \quad$ Line $1 \quad \ldots$ small s $\left(\ll G^{-1}\right) \ldots$

p. 25 Line $10 \ldots$ is no guarantee ...

p. $32 \quad \mathrm{Eq} \cdot(6.12) \quad \ldots \sim \frac{\mathrm{GM}_{\mathrm{W}}^{2} \sigma_{\mathrm{WN}}}{\mathrm{G}^{2} \mathrm{M}_{\mathrm{W}}^{2}} \sim \ldots$

p. 33 Line 2 .. low muon fluxes ...

p. 34 Add "We also thank C. Albright for pointing out an error in the original manuscript."

p. $37 \quad$ Footnote 28 , line $2 \ldots$ as $s \rightarrow \infty \ldots$

Footnote 28 , line $4 \ldots$ fixed singularity at $\mathrm{J}=1$.

p. 38 Replace both minus signs by plus signs

Replace everywhere the word garyon by sthenon 
SLAC-PUB-950

(TH)and(EXP)

August 1971

ON WEAK IN'TERACTIONS AT HIGH ENERGIES ${ }^{\dagger}$

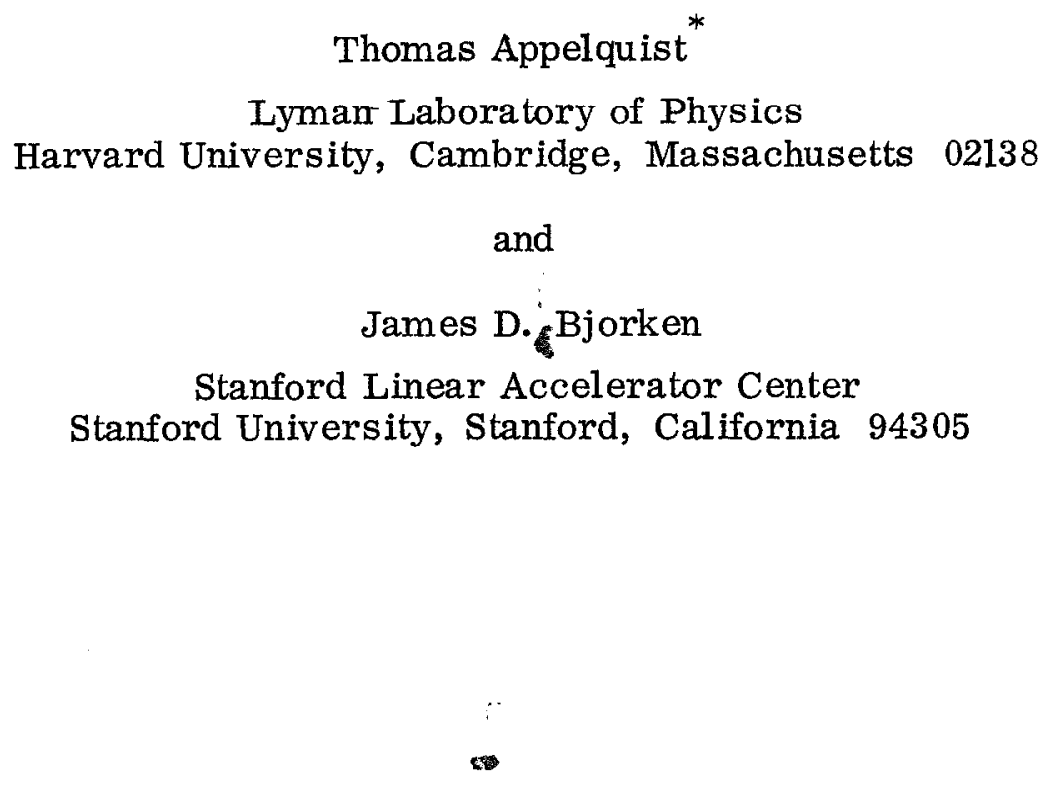

(Submitted to Phys. Rev.)

$\uparrow$ Work supported in part by the U. S. Atomic Energy Commission.

* Work supported in part by Air Force Office of Scientific Research under contract F44620-70-C-0030. 


\begin{abstract}
We discuss the weak interactions from an S-matrix point of view in order to make some qualitative statements about high energy behavior. In order to separate the weak interactions from others as much as possible, attention is focused on the weak interactions of leptons neglecting electromagnetism. We examine the consequences of imposing the constraints of unitarity and analyticity on weak amplitudes whose low energy behavior is assumed to be correctly given by the usual Fermi theory (including the possibility of neutral currents). We first study corrections to the low energy limit by using the Mandelstam interation to express all lepton-lepton two body scattering amplitudes to third order in the Fermi constant in terms of a small number of subtraction constants. We then speculate beyond perturbation theory. We discuss some consequences of forward dispersion relations and propose a mechanism for providing the necessary damping of weak amplitudes at high energies. It is the existence of the intermediate vector boson coupled weakly to leptons but interacting strongly with itself. We examine some consequences of this hypothesis.
\end{abstract}




\section{INTRODUCTION}

The weak interactions as we now know them are without question the low energy limit of a class of phenomena of great richness and variety. The characteristic center-of-mass energy needed for weak processes to reveal

this richness is unknown; it is certainly ${ }^{1,2}$ less than $1000 \mathrm{GeV}$, probably ${ }^{3,4}$ less than $100 \mathrm{GeV}$, perhaps ${ }^{5,6,7}$ as low as $10 \mathrm{GeV}$. At present we are in the position of trying to guess the nature of the whole given only the small fragment available to us at the present superlow energies.

Such a situation is not unprecedented. Strong interaction phenomena, retrospectively viewed from the perspective of the late $1930^{\prime}$ s to the early postwar period, were quite analogous. The symmetry of the nucleon-nucleon force was known, and the Yukawa meson had been postulated to mediate the force, but not yet discovered. However, the theoretical efforts made then to elucidate the nature of the hadron phenomena to come were generally unproductive. Quantum field theory was the only tool available, and, compared to today, in a relatively primitive condition. And, while field theory was generally conceded at that time $^{8}$ not to be "relevant", it still conditioned most attempts to interpret strong interactions.

Much of the progress in hadron physics in the last fifteen years has rested on an attitude less ambitious and more descriptive than one based on a set of coupled local wave equations. S-matrix phenomenology uses general principles to correlate data and exhibit its broad outlines in a qualitatively successful manner. It would be a major advance to have a qualitative picture of weak processes at $1000 \mathrm{GeV}$ center-of-mass energy such as exists for hadron physics. It is reasonable to expect that weak phenomena at such energies will be 
just as messy as hadron phenomena appear to us today, and that when $1000 \mathrm{GeV}$ beams of electrons are made to collide against $1000 \mathrm{GeV}$ neutrino beams, the theorist will again fall back on S-matrix concepts and ideas to describe the data.

In this paper, we try to look at weak interactions from an S-matrix point of view, ${ }^{9}$ as one might view them were such experiments imminent. Our emphasis is more toward the dynamics than toward symmetry principles.

We consider lepton-lepton elastic scattering processes, assuming the low-energy limit is accurately given by the current-current form. We first study the corrections to the low-energy limit. We find, given (a) neglect of lepton mass, (b) helicity conservation, (c) neglect of electromagnetic corrections, (d) $\mu$-e universality, and (e) symmetry under the interchange $\ell \leftrightarrow \nu_{\ell}$, that three invariant amplitudes describe all lepton-lepton two-body scattering processes. We compute from the Mandelstam iteration utilizing analyticity and unitarity ${ }^{10}$ these amplitudes through third order in the Fermi constant in terms of 15 subtraction constants. We do this mainly because the calculations are very easy. We then speculate beyond perturbation theory. From the point of view of S-matrix dynamics, one option with a minimum number of difficulties stands out. It is that of the existence 11,12 of the intermediate boson $\mathrm{W}^{ \pm}$coupled weakly to leptons but interacting strongly ${ }^{13}$ with itself. This option of course includes many proposed models $14,15,16$ of weak interactions. Given this option, one may expect the $\mathrm{W}^{ \pm}$to be members of a large family of new particles which interact strongly with each other, which we call garyons; in particular the W should lie on a Regge-trajectory. Using this picture, we can make some order-of-magnitude estimates of lepton-lepton cross-sections.

In Section 2, we set up the kinematics. Section 3 is devoted to the perturbation theory calculations. We discuss some consequences of forward 
dispersion relations in Section 4, and Section 5 contains speculations which go beyond perturbation theory.

In Section 6, we estimate some cross-sections for high energy lepton and hadron processes using the picture of strongly self-coupled W. 


\section{KINEMATICS AND PRELIMINARIES}

In this section we will establish our notation and list the independent amplitudes necessary to describe all two particle to two particle leptonic weak interactions. Partial wave expansions will be introduced and crossing and unitarity will be discussed.

We will work in the zero mass limit of all the leptons and neglect the effects of electromagnetism. The s-channel is chosen to be that for which the total lepton number $\mathrm{L}=\mathrm{L}_{\mathrm{e}}+\mathrm{L}_{\mu}=2$ and the $\mathrm{t}$-channel is that for which $\mathrm{L}_{\mathrm{e}}=\mathrm{L}_{\mu}=0$. We assume $\mu$ e universality as formally expressed by the existence of the $U(2)$ lepton symmetry in the absence of lepton mass. ${ }^{17}$ Assuming in addition the discrete symmetry e $\leftrightarrow \nu_{\mathrm{e}}, \mu \leftrightarrow \nu_{\mu}$ discussed by Lee, ${ }^{18}$ it is then possible to express all lepton-lepton two particle scattering amplitudes in terms of three such amplitudes which we take to be:

$$
\begin{aligned}
& \mathrm{A}(\mathrm{s}, \mathrm{t}): \nu_{\mathrm{e}} \mathrm{e}^{\mu \rightarrow \mathrm{e} \nu_{\mu}} \\
& \mathrm{B}(\mathrm{s}, \mathrm{t}): \nu_{\mathrm{e}} \nu_{\mu} \rightarrow \nu_{\mathrm{e}} \nu_{\mu} \\
& \mathrm{C}(\mathrm{s}, \mathrm{t}): \nu_{\mathrm{e}}^{\mu \rightarrow \nu} \mathrm{e}^{\mu}
\end{aligned}
$$

Since there is only one helicity amplitude in each channel, it is not necessary to include helicity indices. We will choose our phases such that $\mathrm{A}, \mathrm{B}$ and $\mathrm{C}$ are the helicity amplitudes in each channel. Each amplitude has a kinematic zero at $s=0$ and has the same analyticity properites as the invariant amplitude for spinless, massless particles. The diagrams corresponding to these amplitudes are shown in Figure 1 and we catalogue the other amplitudes in terms of these three in Table 1. 
The amplitudes A, B and $\mathrm{C}$ are dimensionless and normalized such that the s-channel differential cross section for, say, process A is given by ${ }^{19}$

$$
\frac{\mathrm{d} \sigma_{\mathrm{A}}}{\mathrm{dt}}=\frac{4 \pi}{\mathrm{s}} \frac{\mathrm{d} \sigma_{\mathrm{A}}}{\mathrm{d} \Omega}=\frac{1}{16 \pi \mathrm{s}^{2}}|\mathrm{~A}(\mathrm{~s}, \mathrm{t})|^{2}
$$

Under crossing, the same expression holds in the other channels, e.g., in the t-channel

$$
\frac{\mathrm{d} \sigma_{\mathrm{A}}^{-}}{\mathrm{ds}}=\frac{1}{16 \pi \mathrm{t}^{2}}|\mathrm{~A}(\mathrm{~s}, \mathrm{t})|^{2}
$$

In the conventional Fermi theory, $\mathrm{B}=\mathrm{C}=0$, while

$$
\mathrm{A}(\mathrm{s}, \mathrm{t})=\frac{\mathrm{G}}{\sqrt{2}} \overline{\mathrm{u}}\left(\mathrm{p}_{\mathrm{e}}\right) \gamma^{\mu}\left(1-\gamma_{5}\right) \mathrm{u}\left(\mathrm{p}_{\nu_{\mathrm{e}}}\right) \overline{\mathrm{u}}\left(\mathrm{p}_{\nu_{\mu}}\right) \gamma_{\mu}\left(1-\gamma_{5}\right) \mathrm{u}\left(\mathrm{p}_{\mu}\right)
$$

where the spinors are normalized such that

$$
u^{\dagger}(p) u(p)=2 E
$$

Then, up to an arbitrary phase,

$$
A(s, t)=4 \sqrt{2} \text { Gs. }
$$

The conventional Fermi theory can be at most a low energy approximation to the complete dynamical description of $\mathrm{A}, \mathrm{B}$ and $\mathrm{C}$ at all energies. In fact, the possibility that the "netural current" amplitudes $\mathrm{B}$ and $\mathrm{C}$ are comparable to A even at low energies has not been ruled out experimentally. Whatever the actual behavior of $\mathrm{A}, \mathrm{B}, \mathrm{C}$; it is important to point out that the description of all two particle leptonic reactions in terms of at most 3 amplitudes (in the zero mass approximation) is a consequence of quite general considerations and already leads 
to several restrictions on these amplitdues. For example, it is clear from Table 1 that "diagonal" processes such as $\nu_{\mathrm{e}} \mathrm{e} \rightarrow \nu_{\mathrm{e}} \mathrm{e}$ can differ significantly in strength from "non-diagonal" processes such as $\nu_{\mu} \mathrm{e} \rightarrow \mu \nu_{\mathrm{e}}$. This, however, can only happen if $\mathrm{C}(\mathrm{s}, \mathrm{t})$ is appreciable, that is, if "neutral current" reactions such as $\nu_{\mu} \mathrm{e} \rightarrow \nu_{\mu} \mathrm{e}$ are large enough to be measured.

Needless to say, there isn't a great deal of experimental data on leptonic weak reactions. The only reaction which has been directly observed is muon decay. The only other reaction for which there are empirical arguments that it exists is $\nu_{\mathrm{e}} \mathrm{e} \rightarrow \nu_{\mathrm{e}} \mathrm{e}$. Astrophysical arguments ${ }^{20}$ give an upper and lower bound on the effective coupling constant $G_{D}$ for this reaction:

$$
\mathrm{G}_{\mathrm{D}}=10^{0 \pm 2} \mathrm{G}_{\mathrm{F}}
$$

where $G_{F}$ is the Fermi coupling constant. An analysis of CERN data by Steiner ${ }^{21}$ gives a better upper limit on the reaction $\nu_{\mathrm{e}} \mathrm{e} \rightarrow \nu_{\mathrm{e}} \mathrm{e}^{\mathrm{e}}$

$$
G_{D} \leq 6.3 G_{F}
$$

with $90 \%$ confidence. In our formalism, these can be interpreted as bounds on the amplitude $C(s, t)-A(s, u)$ relative to $A(s, t)$. The best bound on this amplitude is that reported by Reines and Gurr ${ }^{22}$ who have looked for the reaction $\bar{\nu}_{\mathrm{e}} \mathrm{e} \rightarrow \bar{\nu}_{\mathrm{e}} \mathrm{e}$ using low energy anti-neutrinos from a fission reactor. They report

$$
G_{D}<2 G_{F}
$$

A reaction which involves only the neutral current amplitude $C(s, t)$ is $\nu_{\mu} \mathrm{e} \rightarrow \nu_{\mu} \mathrm{e}$. An analysis of CERN data by Albright ${ }^{23}$ leads to a bound on the effective neutral coupling constant $G_{0}$ :

$$
\begin{aligned}
& G_{0}<0.6 G_{F} . \\
&-8-
\end{aligned}
$$


These bounds on leptonic weak interactions do not yet rule out the possibility that the amplitudes $\mathrm{B}$ and $\mathrm{C}$ are comparable in magnitude to $\mathrm{A}$. It is clearly very important to attempt to reduce the upper limits on these reactions.

It is important to establish phase conventions since we intend to exploit the constraints of two particle unitarity on the amplitudes. This can be done in terms of the partial wave expansion of the helicity amplitudes according to the prescription of Jacob and Wick. ${ }^{24}$ In the s-channel, the total helicity in the center of mass frame is zero and the partial wave expansion is simply the Legendre expansion. The center of mass scattering angle $\theta_{\mathrm{S}}$ is given by

$$
\cos \theta_{\mathrm{S}}=1+\frac{2 \mathrm{t}}{\mathrm{s}}
$$

and

$$
A(s, t)=16 \pi \sum_{j=0}^{\infty}(2 j+1) a_{j s}(s) P_{j}\left(1+\frac{2 t}{s}\right), \quad \begin{aligned}
& s>0 \\
& t, u<0
\end{aligned}
$$

In the t-channel, the Jacob-Wick expansion takes the form

$$
16 \pi \sum(2 j+1) a_{j t}(t) d_{\lambda \mu}^{j}\left(\theta_{t}\right) e^{i(\lambda-\mu) \phi}
$$

where $\lambda(\mu)$ is the total helicity in the initial (final) state. If the initial direction (positive $\mathrm{z}$ axis) is taken to be that of the incoming anti-lepton, then $\lambda=1$. The phase $e^{i(\lambda-\mu) \phi}$ can be eliminated by choosing the final direction to be that of the outgoing anti-lepton . Then $\mu=1$,

$$
\cos \theta_{t}=1+\frac{2 u}{t}
$$


and

$$
A(s, t)=16 \pi \sum_{j=1}^{\infty}(2 j+1) a_{j t}(t) d_{1 I}^{j}\left(1+\frac{2 u}{t}\right), \quad \begin{array}{r}
t>0 \\
s, u<0
\end{array}
$$

In the u-channel, the same considerations lead to

$$
A(s, t)=16 \pi \sum_{j=1}^{\infty}(2 j+1) a_{j u}(u) d_{11}^{j}\left(1+2 \frac{t}{u}\right), \quad \begin{array}{r}
u>0 \\
s, t<0
\end{array}
$$

With the above conventions, the amplitudes A, B, and C are the helicity amplitudes in each channel. We list here for future reference the Rodrigues formulae and orthogonality properties of the $P_{j}$ and $d_{11}^{j}$.

$$
\begin{gathered}
P_{j}(1-2 x)=d_{00}^{j}(1-2 x)=\frac{1}{j}\left(\frac{d}{d x}\right)^{j} x^{j}(1-x){ }^{j} \\
d_{11}^{j}(1-2 x)=\frac{1}{(j-1) !(1-x)} \frac{d^{j-1}}{d x^{j-1}} x^{j-1}(1-x) \\
\int_{-1}^{+1} d z d_{\mu \nu}^{j}(z) d_{\mu \nu}^{j p}(z)=\frac{2 \delta_{j j^{\prime}}}{2 j+1}
\end{gathered}
$$

The unitarity condition can be expressed by the optical theorem for $\mathrm{B}$ and $\mathrm{C}$, e.g.,

$$
\operatorname{Im} B(s, 0)=s \sigma_{\text {TOT }}^{\nu \nu}(s)
$$

In terms of the partial wave amplitudes,

$$
\operatorname{Im} b_{j s}(s)=\left|b_{j s}(s)\right|^{2}+\text { inel. cont. }
$$

The two particle unitarity relations for all three amplitudes in all channels will 
be tabulated and used in the next section.

It is interesting to consider the consequences of imposing additional symmetry among the lepton amplitudes. An appealing way to do this is to extend the discrete symmetry e $\nleftarrow \nu_{\mathrm{e}}$ plus $\mu \leftrightarrow \nu_{\mu}$ to complete isotopic spin symmetry where $\left(e, \nu_{e}\right)$ and $\left(\mu, \nu_{\mu}\right)$ are taken to be isotopic doublets. The result is that three independent amplitudes are reduced to two corresponding to scattering in the $\mathrm{I}=0$ and $\mathrm{I}=1$ channels. One finds that

$$
A+C=B
$$

and that $A$ and $A+2 C$ are the amplitudes for scattering in the $I=1$ and $I=0$ channels respectively.

Because of the fact that istopic spin imposes no constraints on $A$ and $C$ alone but only relates then to $\mathrm{B}$, it leads to no relations among reactions such as $\nu_{\mu} \mathrm{e} \rightarrow \mu \nu_{\mathrm{e}}, \nu_{\mu} \mathrm{e} \rightarrow \nu_{\mu} \mathrm{e}$ and $\nu_{\mathrm{e}} \mathrm{e} \rightarrow \nu_{\mathrm{e}} \mathrm{e}$ beyond thosc already mentioned. In order to make predictions about these processes alone, one must make additional assumptions. For instance, if the weak current is assumed to be pure isovector, then to lowest order,

$$
\begin{gathered}
\sigma\left(\nu_{\mu} \mathrm{e} \rightarrow \nu_{\mu} \mathrm{e}\right)=\frac{1}{4} \sigma\left(\nu_{\mu} \mathrm{e} \rightarrow \mu \nu_{\mathrm{e}}\right) \\
\sigma\left(\nu_{\mathrm{e}} \mathrm{e} \rightarrow \nu_{\mathrm{e}} \mathrm{e}\right)=\frac{9}{4} \sigma\left(\nu_{\mu} \mathrm{e} \rightarrow \mu \nu_{\mathrm{e}}\right)
\end{gathered}
$$




\section{PERTURBATION THEORY}

One possible way of constructing a phenomenological field theory of weak interactions is to add to the conventional (four fermion or intermediate vector boson) Lagrangian an infinite number of interaction terms to remove the divergences of the conventional theory. ${ }^{25}$ A perturbation expansion in $\mathrm{G} \approx 10^{-5} \mathrm{~m}_{\mathrm{p}}^{-2}$ may then be developed for low energies in terms of arbitrary parameters which mask our ignorance of the true high energy behavior of the theory and which must be determined from experiment. This expansion for, say, the amplitudes A, B and $\mathrm{C}$ will only be good for $s, t, u<\lambda^{2}$, where certainly the radius of convergence $\lambda^{2}<\mathrm{G}^{-1}$ since the effective expansion parameters are Gs and Gt. The closer one gets to this limit the more orders of perturbation theory and hence the more arbitrary constants must be included to fit experiment. A simple way of generating this perturbation expansion for the two particle scattering amplitudes without explicit reference to Lagrangian counter terms is the Mandelstam iteration ${ }^{10}$ using analyticity and unitarity. The general form of the perturbation expansion for $A, B$ and $C$ is

$$
\begin{aligned}
& \mathrm{A}(\mathrm{s}, \mathrm{t})=\sum_{\mathrm{n}, \mathrm{m}} \alpha_{\mathrm{nm}}(\mathrm{Gs})^{\mathrm{n}}(\mathrm{Gt})^{\mathrm{m}}+\text { unitarity } \\
& \mathrm{B}(\mathrm{s}, \mathrm{t})=\sum_{\mathrm{n}, \mathrm{m}} \beta_{\mathrm{nm}}(\mathrm{Gs})^{\mathrm{n}}(\mathrm{Gt})^{\mathrm{m}}+\text { unitarity } \\
& \mathrm{C}(\mathrm{s}, \mathrm{t})=\sum_{\mathrm{n}, \mathrm{m}} \gamma_{\mathrm{nm}}(\mathrm{Gs})^{\mathrm{n}}(\mathrm{Gt})^{\mathrm{m}}+\text { unitarity }
\end{aligned}
$$

The kinematic zero at $\mathrm{s}=0$ means that $\mathrm{n} \geq 1$ while $\mathrm{m} \geq 0$. In the conventional Fermi theory, $\beta_{10}=\gamma_{10}=0$ and $\alpha_{10}=4 \sqrt{2}$. The unitarity contributions which 
have nonvanishing absorptive parts will be specified iteratively in the approximation of keeping only two lepton intermediate states. This will carry us through third order in perturbation theory.

We begin by developing the two particle unitarity relations in terms of the partial wave expansion. In the s-channel, the forward two particle unitarity relations are

$$
\begin{gathered}
\operatorname{Im}_{\mathrm{S}} \mathrm{A}(\mathrm{s}, 0)=\frac{1}{64 \pi^{2}} \int \mathrm{d} \Omega_{\mathrm{S}} 2 \operatorname{Re} \mathrm{A}^{*} \mathrm{C} \\
\operatorname{Im}_{\mathrm{S}} \mathrm{B}(\mathrm{s}, 0)=\frac{1}{64 \pi^{2}} \int \mathrm{d} \Omega_{\mathrm{S}}|\mathrm{B}|^{2} \\
\operatorname{Im}_{\mathrm{S}} \mathrm{C}(\mathrm{s}, 0)=\frac{1}{64 \pi^{2}} \int \mathrm{d} \Omega_{\mathrm{S}}\left[|\mathrm{C}|^{2}+|\mathrm{A}|^{2}\right]
\end{gathered}
$$

In the t-channel, it is convenient to write the two particle unitarity relations in the backward $(u=0)$ direction. Then

$$
\begin{gathered}
\operatorname{Im}_{t} A(-t, t)=\frac{1}{64 \pi^{2}} \int d \Omega_{t} 2 \operatorname{Re} A^{*}(s, t)[A(s, t)-C(s, u)] \\
\operatorname{Im}_{t} B(-t, t)=\frac{1}{64 \pi^{2}} \int d \Omega_{t} 2 \operatorname{Re}\left\{B^{*}(s, t)[B(s, t)-B(s, u)]+C^{*}(s, t)[C(s, t)-A(s, u)]\right\} \\
\operatorname{Im}_{t} C(-t, t)=\frac{1}{64 \pi^{2}} \int d \Omega_{t} 2 \operatorname{Re}\left\{C^{*}(s, t)[B(s, t)-B(s, u)]+B^{*}(s, t)[C(s, t)-A(s, u)]\right\}
\end{gathered}
$$

In the $u$-channel, we take $t=0$ (the forward direction) and

$$
\begin{aligned}
& \operatorname{Im}_{u} A(-u, 0)=\frac{1}{64 \pi^{2}} \int \mathrm{d} \Omega_{\mathrm{u}} 2 \operatorname{Re} \mathrm{A}^{*}(\mathrm{~s}, \mathrm{t}) \mathrm{B}(\mathrm{s}, \mathrm{t}) \\
& \operatorname{Im}_{\mathrm{u}} \mathrm{B}(-\mathrm{u}, 0)=\frac{1}{64 \pi^{2}} \int \mathrm{d} \Omega_{\mathrm{u}}\left[|\mathrm{A}(\mathrm{s}, \mathrm{t})|^{2}+|\mathrm{B}(\mathrm{s}, \mathrm{t})|^{2}\right] \\
& \operatorname{Im}_{\mathrm{u}} \mathrm{C}(-\mathrm{u}, 0)=\frac{1}{64 \pi^{2}} \int \mathrm{d} \Omega_{\mathrm{u}}|\mathrm{C}(\mathrm{s}, \mathrm{t})|^{2}
\end{aligned}
$$


The minus signs in Eq. (3.3) are required by Fermi statistics. Introducing the partial wave expansions, Eq. (2.7) to Eq. (2.9), now leads to the unitarity relations for the partial wave amplitudes

$$
\begin{aligned}
& \operatorname{Im} a_{j s}(s)=2 R e a_{j S}^{*}(s) c_{j s}(s) \\
& \operatorname{Im} b_{j s}(s)=\left|b_{j s}(s)\right|^{2} \\
& \operatorname{Im} c_{j s}(s)_{-}=\left|c_{j s}(s)\right|^{2}+\left|a_{j s}(s)\right|^{2} \\
& \operatorname{Im} a_{j t}(t)=2 R e a_{j t}^{*}(t)\left[a_{j t}(t)-c_{j u}(t)\right] \\
& \operatorname{Im} b_{j t}(t)=2 \operatorname{Re} b_{j t}(t)\left[b_{j t}(t)-b_{j u}(t)\right]+2 \operatorname{Re} c_{j t}^{*}(t)\left[c_{j t}(t)-a_{j u}(t)\right] \\
& \operatorname{Im} c_{j t} f(t)=2 R e b_{j t}^{*}(t)\left[c_{j t}(t)-a_{j u}(t)\right]+2 R e c_{j t}^{*}(t)\left[b_{j t}(t)-b_{j u}(t)\right] \\
& \operatorname{Im} a_{j u}(u)=2 \operatorname{Re} a_{j u}^{*}(u) b_{j u}(u) \\
& \operatorname{Im} b_{j u}(u)=\left|b_{j u}(u)\right|^{2}+\left|a_{j u}(u)\right|^{2} \\
& \operatorname{Im} c_{j u}(u)=\left|c_{j u}(u)\right|^{2}
\end{aligned}
$$

With the above apparatus, it is relatively easy to carry out the perturbation expansion to third order. In first order

$$
\mathrm{A}=\alpha_{10} \mathrm{Gs} \quad \mathrm{B}=\beta_{10} \mathrm{Gs} \quad \mathrm{C}=\gamma_{10} \mathrm{Gs}
$$


and the nonvanishing partial wave amplitudes are

$$
\begin{array}{lll}
16 \pi \mathrm{a}_{0 \mathrm{~s}}=\alpha_{10} \mathrm{Gs} & 48 \pi \mathrm{a}_{1 \mathrm{t}}=-\alpha_{10} \mathrm{Gt} & 48 \pi \mathrm{a}_{1 \mathrm{u}}=-\alpha_{10} \mathrm{Gu} \\
16 \pi \mathrm{b}_{0 \mathrm{~s}}=\beta_{10} \mathrm{Gs} & 48 \pi \mathrm{b}_{1 \mathrm{t}}=-\beta_{10} \mathrm{Gt} & 48 \pi \mathrm{b}_{1 \mathrm{u}}=-\beta_{10} \mathrm{Gu} \\
16 \pi \mathrm{c}_{0 \mathrm{~s}}=\gamma_{10} \mathrm{Gs} & 48 \pi \mathrm{c}_{1 \mathrm{t}}=\gamma_{10} \mathrm{Gt} & 48 \pi \mathrm{c}_{1 \mathrm{u}}=-\gamma_{10} \mathrm{Gu}
\end{array}
$$

Thus to second order

$$
\begin{aligned}
& (16 \pi)^{2} \operatorname{Im} a_{0 s}=2 \alpha_{10} \gamma_{10}(\mathrm{Gs})^{2} \\
& (16 \pi)^{2} \operatorname{Im} b_{0 s}=\beta_{10}{ }^{2}(\mathrm{Gs})^{2} \\
& (16 \pi)^{2} \operatorname{Im} \mathrm{c}_{0 \mathrm{~s}}=\left(\gamma_{10}{ }^{2}+\alpha_{10}{ }^{2}\right)(\mathrm{Gs})^{2} \\
& (48 \pi)^{2} \operatorname{Im} \mathrm{a}_{1 \mathrm{t}}=2 \alpha_{10}\left(\alpha_{10}-\gamma_{10}\right)(\mathrm{Gt})^{2} \\
& (48 \pi)^{2} \operatorname{Im} b_{1 \mathrm{t}}=2 \gamma_{10}\left(\gamma_{10}-\alpha_{10}\right)(\mathrm{Gt})^{2} \\
& (48 \pi)^{2} \operatorname{Im} c_{1 \mathrm{t}}=2 \beta_{10}\left(\gamma_{10}-\alpha_{10}\right)(\mathrm{Gt})^{2} \\
& (48 \pi)^{2} \operatorname{Im} \mathrm{a}_{1 \mathrm{u}}=2 \alpha_{10} \beta_{10}(\mathrm{Gu})^{2} \\
& (48 \pi)^{2} \operatorname{Im} b_{1 \mathrm{u}}=\left(\beta_{10}{ }^{2}+\alpha_{10}\right)_{(\mathrm{Gu})}^{2} \\
& (48 \pi)^{2} \operatorname{Im} \mathrm{c}_{1 \mathrm{u}}=\gamma_{10}{ }^{2}(\mathrm{Gu})^{2}
\end{aligned}
$$

The second order amplitudes can now be consturcted using the dispersion relations and performing the necessary subtractions. An imaginary part of the form (Gx) ${ }^{2}$ corresponds to a function of the form $-1 / \pi(G x){ }^{2} \log (-G x)$. The 
amplitudes through second order in perturbation theory are

$$
\begin{aligned}
& \mathrm{A}(\mathrm{s}, \mathrm{t})=\alpha_{10} \mathrm{Gs}+\alpha_{20}(\mathrm{Gs})^{2}+\alpha_{11} \mathrm{GsGt}-\frac{\alpha_{10} \gamma_{10}}{8 \pi^{2}}(\mathrm{Gs})^{2} \log (-\mathrm{Gs}) \\
& +\frac{\alpha_{10}\left(\alpha_{10}-\gamma_{10}\right)}{24 \pi^{2}} \text { GsGt } \log (-\mathrm{Gt})+\frac{\alpha_{10} \gamma_{10}}{24 \pi^{2}} \text { GsGu } \log (-\mathrm{Gu}) \\
& \mathrm{B}(\mathrm{s}, \mathrm{t})=\beta_{10} \mathrm{Gs}+\beta_{20}(\mathrm{Gs})^{2}=\beta_{11} \mathrm{GsGt}-\frac{\beta_{10}^{2}}{16 \pi^{2}}(\mathrm{Gs})^{2} \log (-\mathrm{Gs}) \\
& +\frac{\gamma_{10}\left(\gamma_{10^{-\alpha}}\right)}{24 \pi^{2}} \text { GsGt } \log (-\mathrm{Gt})+\frac{\alpha_{10^{2}+\beta_{10}^{2}}}{48 \pi^{2}} \text { GsGu } \log (-\mathrm{Gu}) \\
& \mathbf{C}(\mathrm{s}, \mathrm{t})=\gamma_{10} \mathrm{Gs}+\gamma_{20}(\mathrm{Gs})^{2}+\gamma_{11} \mathrm{GsGt}-\frac{\alpha_{10^{2}+\gamma_{10}{ }^{2}}}{16 \pi^{2}} \text { (Gs) }{ }^{2} \log (-\mathrm{Gs}) \\
& +\frac{\beta_{10}\left(\gamma_{10}-\alpha_{10}\right)}{24 \pi^{2}} \text { GsGt } \log (-\mathrm{Gt})+\frac{\gamma_{10}^{2}}{48 \pi^{2}} \text { GsGu } \log (-\mathrm{Gu})
\end{aligned}
$$

All the parameters must be determined by experiment. From $\mu$ decay, it is known that $\alpha_{10}=4 \sqrt{2}$ and that $\alpha_{20}$ and $\alpha_{11}$ are certainly much less than $10^{5}$. As discussed in Section 2, there exist upper limits on $\beta_{10}$ and $\gamma_{10}$ but they could be the same order of magnitude as $\alpha_{10}$.

Since the machinery set up here makes these perturbative calculations so simple, we can't resist pushing on to third order. To keep things simple, we restrict ourselves to the case of the conventional Fermi theory:

$$
\alpha_{10}=4 \sqrt{2} \quad, \quad \beta_{10}-\gamma_{10}=0
$$


Then the second order results above become

$$
\begin{aligned}
& \mathrm{A}(\mathrm{s}, \mathrm{t})=4 \sqrt{2} \mathrm{Gs}+\alpha_{20}(\mathrm{Gs})^{2}+\alpha_{11} \mathrm{GsGt}+\frac{4}{3 \pi^{2}} \mathrm{GsGt} \log (-\mathrm{Gt}) \\
& \mathrm{B}(\mathrm{s}, \mathrm{t})=\beta_{20}(\mathrm{Gs})^{2}+\beta_{11} \mathrm{GsG}+\frac{2}{3 \pi^{2}} \mathrm{GsGu} \log (-\mathrm{Gu}) \\
& \mathrm{C}(\mathrm{s}, \mathrm{t})=\gamma_{20}(\mathrm{Gs})^{2}+\gamma_{11} \mathrm{GsG}-\frac{2}{\pi^{2}}(\mathrm{Gs})^{2} \log (-\mathrm{Gs})
\end{aligned}
$$

We first project out the partia $\bar{l}$ wave amplitudes in each channel. Since only terms up to third order will be kept in the unitarity relations (3.5) to (3.7), the only partial wave amplitudes we need are

$$
\begin{aligned}
& 16 \pi \mathrm{a}_{0 \mathrm{~s}}=4 \sqrt{2} \mathrm{Gs}+\alpha_{20}-\frac{1}{2} \alpha_{11}(\mathrm{Gs})^{2}-\frac{4}{3 \pi^{2}}(\mathrm{Gs})^{2}\left(\frac{1}{2} \log \mathrm{Gs}-\frac{1}{4}\right) \\
& 16 \pi \mathrm{b}_{0 \mathrm{~s}}=\left(\beta_{20}-\frac{1}{2} \beta_{11}\right)(\mathrm{Gs})^{2}-\frac{2}{3 \pi^{2}}(\mathrm{Gs})^{2}\left(\frac{1}{2} \log \mathrm{Gs}-\frac{1}{4}\right) \\
& 16 \pi \mathrm{c}_{0 \mathrm{~s}}=\left(\gamma_{20}-\frac{1}{2} \gamma_{11}\right)(\mathrm{Gs})^{2}-\frac{2}{\pi^{2}}(\mathrm{Gs})^{2} \log (-\mathrm{Gs}) \\
& 16 \pi \mathrm{a}_{1 \mathrm{t}}=-\frac{4 \sqrt{2}}{3} \mathrm{Gt}+\left(\frac{1}{4} \alpha_{20}-\frac{1}{3} \alpha_{11}\right)(\mathrm{Gt})^{2}-\frac{4}{9 \pi^{2}}(\mathrm{Gt})^{2} \log (-\mathrm{G} \mathrm{t}) \\
& 16 \pi \mathrm{b}_{1 \mathrm{t}}=\left(\frac{1}{4} \beta_{20}-\frac{1}{3} \beta_{11}\right)(\mathrm{Gt})^{2}+\frac{2}{3 \pi^{2}}(\mathrm{Gt})^{2}\left(\frac{1}{12} \log \mathrm{Gt}-\frac{13}{144}\right) \\
& 16 \pi c_{1 \mathrm{t}}=\left(\frac{1}{4} \gamma_{20}-\frac{1}{3} \gamma_{11}\right)(\mathrm{Gt})^{2}-\frac{2}{\pi^{2}}(\mathrm{Gt})^{2}\left(\frac{1}{4} \log \mathrm{Gt}-\frac{1}{16}\right) \\
& 16 \pi \mathrm{a}_{1 \mathrm{u}}=-\frac{4 \sqrt{2}}{3} \mathrm{Gu}+\frac{1}{4}\left(\alpha_{20}+\frac{1}{12} \alpha_{11}\right)(\mathrm{Gu})^{2}+\frac{4}{3 \pi^{2}}(\mathrm{Gu})^{2}\left(\frac{1}{12} \log \mathrm{Gu}-\frac{13}{144}\right) \\
& 16 \pi \mathrm{b}_{1 \mathrm{u}}=\left(\frac{1}{4} \beta_{20}+\frac{1}{12} \beta_{11}\right)(\mathrm{Gu})^{2}-\frac{2}{9 \pi^{2}}(\mathrm{Gu})^{2} \log (-\mathrm{Gu}) \\
& 16 \pi c_{1 u}=\left(\frac{1}{4} \gamma_{20}+\frac{1}{12} \gamma_{11}\right)(\mathrm{Gu})^{2}-\frac{2}{\pi^{2}}(\mathrm{Gu})^{2}\left(\frac{1}{4} \log \mathrm{Gu}-\frac{1}{16}\right)
\end{aligned}
$$


The unitarity relations (3.5) to (3.7) give the absorptive parts of the partial wave amplitudes through third order. The amplitudes $A, B$ and $C$ can then be constructed as before. In the s-channel, for example, an absorptive part of the form (Gs) ${ }^{\mathrm{n}}$ corresponds to the contribution $-1 / \pi(\mathrm{gs}){ }^{\mathrm{n}} \log (-\mathrm{Gs})$ to the unitarity part of the amplitude. An absorptive part of the form $(\mathrm{Gs}) \mathrm{n}_{\log G s}$ corresponds to a term in the amplitude of the form $-1 / 2 \pi(\mathrm{gs}) \mathrm{n} \log ^{2}$ (-Gs). The amplitudes through third order are

$$
\begin{aligned}
\mathrm{A}(\mathrm{s}, \mathrm{t})= & 4 \sqrt{2} \mathrm{Gs}+\sum_{\substack{\mathrm{n}+\mathrm{m}=2,3 \\
\mathrm{~m} \geq 1}} \alpha_{\mathrm{nm}}(\mathrm{Gs})^{\mathrm{n}}(\mathrm{Gt})^{\mathrm{m}}+\frac{4}{3 \pi^{2}} \mathrm{GsGt} \log (-\mathrm{Gt}) \\
& -\frac{\sqrt{2}}{2 \pi^{2}}(\mathrm{Gs})^{3} \log (-\mathrm{Gs})\left[\gamma_{20}-\frac{1}{2} \gamma_{11}-\frac{1}{\pi^{2}} \log (-\mathrm{Gs})\right] \\
& -\frac{\sqrt{2}}{2 \pi^{2}} \mathrm{Gs}(\mathrm{Gt})^{2} \log (-\mathrm{Gt})\left[\frac{1}{2} \alpha_{20}-\frac{2}{3} \alpha_{11}-\frac{1}{4} \gamma_{20}-\frac{1}{12} \gamma_{11}-\frac{1}{8 \pi^{2}}-\frac{7}{36 \pi^{2}} \log (-\mathrm{Gt})\right] \\
& -\frac{\sqrt{2}}{2 \pi^{2}} \mathrm{Gs}(\mathrm{Gu})^{2} \log (-\mathrm{Gu})\left[\frac{1}{4} \beta_{20}+\frac{1}{12} \beta_{11}-\frac{1}{9 \pi^{2}} \log (-\mathrm{Gu})\right] \\
\mathrm{B}(\mathrm{s}, \mathrm{t})= & \sum_{\mathrm{n}+\mathrm{m}=2,3} \beta_{\mathrm{nm}}(\mathrm{Gs})^{\mathrm{n}(\mathrm{Gt})} \mathrm{m}+\frac{2}{3 \pi^{2}} \mathrm{GsGu} \log (-\mathrm{Gu}) \\
& \mathrm{m} \geq 17) \\
& +\frac{\sqrt{2}}{2 \pi^{2}} \mathrm{Gs}(\mathrm{Gt})^{2} \log (-\mathrm{Gt})\left[\frac{1}{4} \gamma_{20}-\frac{1}{3} \gamma_{11}+\frac{1}{8 \pi^{2}}-\frac{1}{4 \pi^{2}} \log (-\mathrm{Gt})\right] \\
& \left.-\frac{\sqrt{2}_{2}^{2} \mathrm{Gs}(\mathrm{Gu})^{2} \log (-\mathrm{Gu})\left[\frac{1}{4} \alpha_{20}+\frac{1}{12} \alpha_{11}-\frac{13}{108 \pi^{2}}+\frac{1}{18 \pi^{2}} \log (-\mathrm{Gu})\right]}{2 \pi^{2}}\right]
\end{aligned}
$$




$$
\begin{aligned}
C(s, t)= & \sum_{\substack{n+m=0,3 \\
n \geq 1}} \gamma_{n m}(G s)^{n}(G t)^{m}-\frac{2}{\pi^{2}}(G s)^{2} \log (-G s) \\
& -\frac{\sqrt{2}}{2 \pi^{2}}(G s)^{3} \log (-G s)\left[\alpha_{20}-\frac{1}{2} \alpha_{11}+\frac{1}{3 \pi^{2}}-\frac{1}{3 \pi^{2}} \log (-G s)\right] \\
& +\frac{\sqrt{2}}{2 \pi^{2}} \operatorname{Gs}(G t)^{2} \log (-G t)\left[\frac{1}{4} \beta_{20}-\frac{1}{3} \beta_{11}-\frac{13}{216 \pi^{2}}+\frac{1}{36 \pi^{2}} \log (-G t)\right]
\end{aligned}
$$

Thus with the assignments (3.12), there are 15 additional parameters necessary to describe all two particle leptonic weak interactions through third order in perturbation theory.

The perturbation expasnion has been developed including only leptonic intermediate states. This is not a very good approximation since the values of $s$ and $t$ at which higher order terms in the expansion become important are probably much greater than typical hadronic masses. Through third order in the perturbation expansion, hadronic intermediate states enter only in the t-channel unitarity relation where $\mathrm{L}_{\mathrm{e}}=\mathrm{L}_{\mu}=0$. They will give absorptive parts leading to dispersion integrals which we assume require no more subtractions than those arising from lepton-antilepton pair intermediate states. There will thus be hadronic contributions to the subtraction constants $\alpha_{\mathrm{nm}}, \beta_{\mathrm{mn}}$ and $\gamma_{\mathrm{nm}}$. If we ask the perturbation expansion to have a radius of convergence large compared to $1 \mathrm{GeV}$, there will also be pole and cut contributions determined by the details of the strong interactions. It is to be emphasized that unlike the subtraction constants $\alpha_{\mathrm{nm}}, \beta_{\mathrm{nm}}$ and $\gamma_{\mathrm{nm}}$, these coefficients are in principle calculable.

If some possible intermediate state is not explicitly included, then the perturbation expansion will break down as the threshold for the process is 
approached. Consequently, the subtraction constants will not be of order unity. This can be seen by considering the contribution to the process

$$
\nu_{\mathrm{e}}+\mu \rightarrow \mathrm{e}+\nu_{\mu}
$$

coming from $\rho$-exchange. The contribution has the form

$$
\delta \mathrm{A}=c \mathrm{G}^{2} \mathrm{~s}\left(\frac{\mathrm{m}_{\rho}^{4}}{\mathrm{~m}_{\rho}^{2}-\mathrm{t}}\right)
$$

where $\mathrm{c}$ is a number of order unity. Upon expanding in a power series in $t$, we find, comparing with (3.17), that

$$
\begin{aligned}
& \delta \mathrm{A}=\sum_{\mathrm{m}} \delta \alpha_{1 \mathrm{~m}}(\mathrm{Gs})(\mathrm{Gt})^{\mathrm{m}} \\
& \delta_{1 \mathrm{~m}}={\frac{\mathrm{c}}{\left(\mathrm{Gm} \mathrm{m}^{2}\right)}}^{\mathrm{m}-1}
\end{aligned}
$$

This of course results from the small radius of convergence $\mathrm{m}_{\rho}^{2}$ for the expansion in powers of $t$. One must keep in mind that the same phenomenon will occur if the important range of interaction $\lambda^{-2}$ for weak processes is large compared with the Fermi constant G. 


\section{DISPERSION RELATIONS AND SUM RULES}

In this section we briefly examine some consequences of the assumption that the lepton-lepton amplitudes A, B, C satisfy dispersion relations in $\mathrm{s}$ for fixed $t$. We shall assume, in analogy with strong interactions, no more than two subtractions are necessary at $t=0$. Our discussion shall be restricted to forward-scattering dispersion relations. With further assumptions about the existence of $d^{n} A(s, t) / d t_{n}$ at $\bar{t}=0$, more can be said, ${ }^{26}$ but we shall not go into this here.

We will write down a sequence of dispersion relations sum rules which we will use to make some order of magnitude statements about the weak interaction "cutoff" $\lambda^{2}$.

Consider first the amplitude

$$
D(s, t)=C(s, t)-A(s, u)
$$

for the process $\nu_{e} e^{\rightarrow} \nu_{e} e$. Assuming the validity of the Pomeranchuk theorem 27

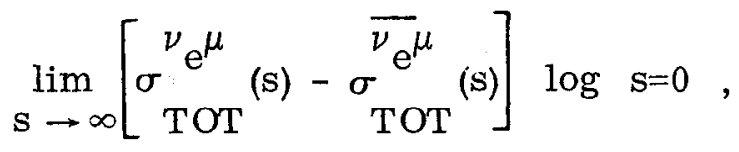

one may write a once subtracted dispersion relation at $\mathrm{t}=0 .{ }^{28}$

$$
\mathrm{D}(\mathrm{s}, 0)=\frac{\mathrm{s}}{\pi} \int_{0}^{\infty} \mathrm{d} \mathrm{s}^{\prime}\left[\frac{\sigma^{\nu \mathrm{e}}\left(\mathrm{s}^{\prime}\right)}{\mathrm{TOT}^{\top}-\mathrm{s}}-\frac{\sigma^{\bar{\nu} \mathrm{TOT}}\left(\mathrm{s}^{\prime}\right)}{\mathrm{s}^{\prime}+\mathrm{s}}\right]
$$

Using the low energy theorem coming from first order perturbation theory:

$$
\lim _{\mathrm{s} \rightarrow 0} \frac{\mathrm{D}(\mathrm{s}, 0)}{\mathrm{s}}=\left(\gamma_{10}-\alpha_{10}\right) \mathrm{G}=\frac{1}{\pi} \int_{0}^{\infty} \frac{\mathrm{d} \mathrm{s}}{\mathrm{s}}\left[\begin{array}{cc}
\nu \mathrm{e} & \overline{\nu \mathrm{e}} \\
\sigma_{\mathrm{TOT}} & (\mathrm{s})-\sigma_{\mathrm{TOT}} \\
(\mathrm{s})
\end{array}\right]
$$


Similar sum rules can be written relating the other first order subtraction constants $\gamma_{10} \mathrm{G}$ and $\beta_{10} \mathrm{G}$ to integrals over total cross sections. Since $\alpha=4 \sqrt{2}$ and $\gamma_{10}$ is not greater than this, we have

$$
\int_{0}^{\infty} \frac{\mathrm{ds}}{\mathrm{s}}\left|\sigma_{\mathrm{TOT}}^{\nu \mathrm{e}}(\mathrm{s})-\sigma_{\mathrm{TOT}}^{\overline{\nu e}}(\mathrm{~s})\right| \gtrsim 4 \pi \mathrm{G} \sqrt{2} \approx 7 \times 10^{-32} \mathrm{~cm}^{2}
$$

This implies a sizeable value for either the $\nu_{e} e$ or $\bar{\nu}_{e} e$ total cross section in some region of $\mathrm{s}$.

If the usual charged current picture is correct at low energies $\left(\beta_{10}=\gamma_{10} \ll 1\right)$, the sum rule

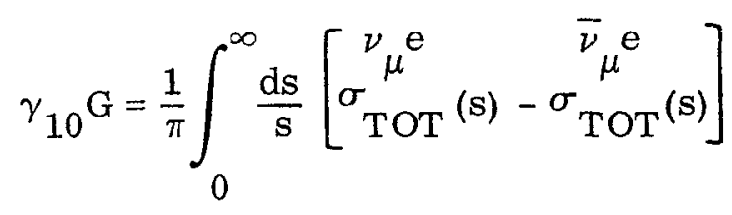

can be used to argue that $\lambda^{2} \leqslant G^{-1}$. In this case the integrand for $s \lesssim \lambda^{2}$ is dominated by the contribution

$$
\sigma_{\mu}^{\nu_{\mu} \mathrm{e} \rightarrow \mu \nu_{\mathrm{e}}}(\mathrm{s}) \approx \frac{1}{16 \pi^{2}}|4 \sqrt{2} \mathrm{Gs}|^{2}=\frac{2 \mathrm{G}^{2} \mathrm{~s}}{\pi}
$$

Thus

$$
\gamma_{10} \approx \frac{2}{\pi^{2}}\left(\mathrm{G} \lambda^{2}\right)+\mathrm{G}^{-1} \int_{\lambda^{2}}^{\infty} \frac{\mathrm{ds}}{\mathrm{s}}\left[\begin{array}{cc}
\nu_{\mu}^{\mathrm{e}} & \bar{\nu}_{\mu}^{\mathrm{e}} \\
\sigma_{\mathrm{TOT}}^{(\mathrm{s})}-\sigma_{\mathrm{TOT}}^{(\mathrm{s})}
\end{array}\right]
$$

and since $\gamma_{10} \ll 1, \lambda^{2} \ll G^{-1}$ unless the residual high energy part of the dispersion integral cancels the low energy part. It is hard on dynamical grounds to see why such a cancellation should occur and there is no symmetry principle 
(such as PCAC or guage invariance) in sight to provide a low energy theorem. This argument can be repeated for the $u=0$ dispersion relation in $t$ for the $\mathrm{C}$ amplitude. For small $\mathrm{t}$

$$
C(-t, t)=-\gamma_{10} G t \quad \frac{t}{\pi} \int_{-\infty}^{+\infty} \frac{d t^{\prime}}{t^{2}} \operatorname{Im} C\left(-t^{\dagger}, t^{\dagger}\right)
$$

Again the contribution to $\gamma_{10}$ form $\left|t^{\varphi}\right|<\lambda^{2}$ is $2\left(\mathrm{G} \lambda^{2} / \pi^{2}\right)$, while the remainder is related to integrals over the absorptive parts of the charge-exchange amplitudes $\bar{\nu}_{\mu}+\nu_{\mu} \rightarrow \mathrm{e}^{+}+\mathrm{e}^{-}$and $\mathrm{e}^{-}+\nu_{\mu} \rightarrow \nu_{\mu}+\mathrm{e}^{-}$at $\mathrm{u}=0$. It is especially plausible that these amplitudes are small enough at high energies to make (4.9) converge. If unitarity considerations are important asymptotically, the charge exchange processes must compete with all other channels open to $\begin{aligned} \bar{\nu}_{\mu}+\nu_{\mu} & \text { and } \mathrm{e}^{-}+\nu_{\mu^{*}} \\ & \text { Just as } \alpha_{10}, \beta_{10} \text { and } \gamma_{10} \text { can be related to integrals over total }\end{aligned}$ cross sections, the higher subtraction constants can also be related to other integrals over total cross sections. Consider, for example, the $\mathrm{C}$ amplitude. From (3.11), the low energy behavior if

$$
\begin{gathered}
\mathrm{C}(\mathrm{s}, 0)=\gamma_{10} \mathrm{Gs}+\gamma_{20}(\mathrm{Gs})^{2}-\frac{\alpha_{10}^{2}+\gamma_{10}^{2}}{16 \pi^{2}}(\mathrm{Gs})^{2} \log (-\mathrm{Gs}) \\
-\frac{\gamma_{10}{ }^{2}(\mathrm{Gs})^{2} \log \mathrm{Gs}}{48 \pi^{2}}+0\left[(\mathrm{Gs})^{3} \log ^{2} \mathrm{Gs}\right]
\end{gathered}
$$

To get a sum rule for $\gamma_{20}$, we can use this along with the twice subtracted dispersion relation

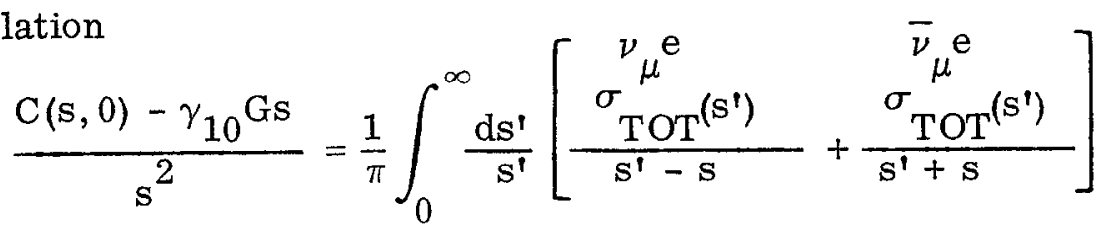


For small $\mathrm{s}\left(\mathrm{G}^{-1}\right)$ the logarithmic unitarity contributions in $(4.10)$ can be written as dispersion integrals, e.g.,

$$
-\frac{\alpha_{10}{ }^{2}+\gamma_{10}{ }^{2}}{16 \pi^{2}}(\mathrm{Gs})^{2} \log (-\mathrm{Gs})=\frac{\mathrm{s}^{2}}{\pi} \int_{0}^{\mathrm{G}^{-1}} \frac{\mathrm{ds}^{\prime} \sigma_{0}^{\nu} \mu^{\mathrm{e}}\left(\mathrm{s}^{\prime}\right)}{\mathrm{s}^{\prime}\left(\mathrm{s}^{\dagger}-\mathrm{s}\right)}+0\left[(\mathrm{Gs})^{3}\right]
$$

where

$$
\sigma_{0}^{\nu} \mu^{\mathrm{e}}(\mathrm{s})=\frac{1}{16 \pi \mathrm{s}^{-}}\left[\alpha_{10}{ }^{2}(\mathrm{Gs})^{2}+\gamma_{10}{ }^{2}(\mathrm{Gs})^{2}\right]
$$

is the contribution to $\sigma_{\mathrm{TOT}}^{\nu_{\mu} \mathrm{e}}\left(\mathrm{s}^{\prime}\right)$ from the first order perturbation theory. Putting (4.10) and (4.12) into (4.11) and letting $s \rightarrow 0$, we have

$$
\begin{aligned}
& \mathrm{G}^{2} \gamma_{20}=\frac{1}{\pi} \int_{0}^{\mathrm{G}^{-1}} \frac{\mathrm{ds}}{\mathrm{s}^{2}}\left\{\left[\begin{array}{c}
\nu_{\mu}^{\mathrm{e}} \\
\sigma_{\mathrm{TOT}}(\mathrm{s})-\sigma_{0}^{\nu_{\mu}}{ }^{\mathrm{e}}(\mathrm{s})
\end{array}\right]+\left[\begin{array}{c}
\bar{\nu}_{\mu}^{\mathrm{e}} \\
\sigma_{\mathrm{TOT}} \bar{\nu}_{\mu} \mathrm{e} \\
\sigma_{0}{ }^{\mathrm{e}}(\mathrm{s})
\end{array}\right]\right\} \\
& +\frac{1}{\pi} \int_{\mathrm{G}^{-1}}^{\infty} \frac{\mathrm{ds}}{\mathrm{s}^{2}}\left[\begin{array}{c}
\nu_{\mu} \mathrm{e} \\
\sigma_{\mathrm{TOT}}^{\mathrm{e}}(\mathrm{s})+\sigma_{\mathrm{TOT}}^{(\mathrm{s})}
\end{array}\right]
\end{aligned}
$$

Similar sum rules can be written for $\gamma_{\mathrm{m} 0}$, but require perturbation theory to be carried to the appropriate order of approximation.

The sum rule (4.14) is on better footing than (4.3) or (4.6) since the assumption of convergence is not as strong. Such higher moment sum rules are however not as useful for constraining the high energy behavior of the cross sections since the extra damping makes them less sensitive to high energy behavior and since the higher subtraction constants are not known. If it is assumed that $\gamma_{20} \lesssim 1$, than since the first integral in (4.14) gives a 
contribution of order $\mathrm{G}^{2}$, a bound of the form

$$
\int_{G^{-1}}^{\infty} \mathrm{ds} \frac{\sigma_{\text {TOT }}^{\nu}{ }^{(s)}}{s^{2}} \leq G^{2}
$$

can be derived. If $\sigma_{\text {TOT }}^{\nu_{\mu}^{e}}$ flattens out (apart from logarithms) to $\sigma_{\infty}$ beyond $\mathrm{s}=\Lambda^{2},(4.15)$ leads to

$$
\sigma_{\text {TOT }}^{\nu_{\mu} \mathrm{e}}(\infty) \leq \mathrm{G}\left(\mathrm{G} \Lambda^{2}\right)
$$

Pomeranchuk, in a most interesting paper published posthumously by his colleagues, ${ }^{26}$ has studied such implications of forward dispersion relations on the minimum energy $s$ for which the lepton-lepton cross-sections can become constant and found essentially (4.16). In addition, he found even stronger restrictions by studying dispersion-relations for derivatives of the forward scattering amplitude. Unless one assumes the stripless approximation discussed in Section 5 , there is not guarantee the derivatives exist, since there is a $t$-channel cut at $t=0$ coming from neutrino-pair exchange. However, arguments can be given ${ }^{26}$ that the first derivative should exist. To the extent such dispersion relations for derivatives of the forward amplitude exist, one can also extend the sum rules, such as (4.14), for $\gamma_{\mathrm{m} 0}$ to other $\gamma_{\mathrm{mn}}$, relating the coefficients to absorptive parts which are positive, because $\left.\frac{d^{n} A(s, t)}{d t^{n}}\right|_{t=0}>0$. But in any case, it is clear that just from fixed $t=0, u=0$, and $s=0$ dispersion relations for $\mathrm{A}, \mathrm{B}$, and $\mathrm{C}$, the coefficients $\alpha_{i j}, \beta_{i j}, \gamma_{i j}(i+j \leq 2)$ can all be determined (and in some cases overdetermined) in terms of integrals over absorptive parts of physical scattering amplitudes. 


\section{BEYOND PERT URBATION T HEORY}

An S-matrix approach to high-energy weak processes should be capable of making progress beyond considerations of a perturbation expansion, as has been done in hadron physics. We are thinking here of the successful use of dispersion relations, of high energy limiting theorems such as the Froissart bound ${ }^{29}$ and the work of Martin, ${ }^{30}$ of the bootstrap concepts and perhaps even of duality. But typically in hadron physics it is difficult to make predictions using S-matrix concepts until a great deal of data exists. We too cannot claim any better, but we shall, for what they are worth, give a natural succession of hypotheses which from the point of view of S-matrix dynamics appears to present the path of least resistance and which lead to some general consequences:

Hypothesis 1: The Mandelstam double-spectral function in the strip regions $\left(0<t<\lambda^{2} ; \quad s\right.$ arbitrary + permutations $\left.s \rightarrow t \rightarrow u\right)$ may be neglected for some $\lambda^{2}$, where $\mathrm{m}^{2}$ (lepton) $<<\lambda^{2} \ll \mathrm{G}^{-1}$ and probably $\lambda^{2}>\mathrm{m}^{2}$ (hadron). This means that asymptotic weak lepton-lepton amplitudes are not controlled by lepton-pair exchange but by exchange of heavy objects such as the intermediate boson $\mathrm{W}$. The alternative to this stripless approximation has the difficulty that one must show that the large spectral-function in the strips does not contribute via fixed-t dispersion relations to low-energy weak processes. Such large lowenergy contributions could ruin the simple current-current picture.

A major consequence of the stripless approximation is the Froissart bound $^{29}$ :

$$
\sigma_{\mathrm{TOT}}(\mathrm{s}) \lesssim \frac{1}{\lambda^{2}}\left[0(1) \log ^{2} \mathrm{~s}\right]
$$


Hypothesis 2: In all parital waves, the partial wave amplitudes $a_{J}$ are always small, $\left|a_{J}\right| \ll 1$, except possibly at positions of some narrow resonances. This is suggested, but not required, by Hypothesis 1 . It again serves to protect reactive effects associated with unitarity requirements from affecting the current-current picture at low energy.

Hypothesis 3: There exist intermediate vector bosons $\mathrm{W}^{ \pm}$with mass $\mathrm{m}_{\mathrm{W}}$ "small", $\mathrm{m}_{\mathrm{W}} \ll 10^{3} \mathrm{GeV}$, and certainly $\mathrm{m}_{\mathrm{W}} \gtrsim \lambda$. This hypothesis, in lowest order, is compatible with the preceding ones and of course is strongly motivated by the current-current picture. However, as emphasized by Gell-Mann, Goldberger, Kroll and Low, ${ }^{6}$ the process

$$
\ell+\bar{l} \rightarrow \mathrm{W}^{+}+\mathrm{W}^{-}
$$

calculated in the OLE (one-lepton-exchange) approximation violates Hypothesis 2 at sufficiently high energies, and the easiest way out, requiring no miracles, is the next hypothesis.

Hypothesis 4: There exist strong interactions of $\mathrm{w}^{ \pm}$with each other but not with leptons. This latter proviso ensures again that the low-energy current-current picture is not modified by W-lepton rescattering effects such as shown in Figure 2.

The effect of the strong W-W coupling on the reaction (5.2) is to provide strong damping of the OLE contribution at high energies. This is most convincingly seen by looking at the reaction in the opposite direction: $\mathrm{W}^{+}+\mathrm{W}^{-} \rightarrow \ell+\bar{\ell}$. The final $\ell \bar{l}$ channel competes poorly with many other open channels composed of particles in the strongly coupled $W$ family. It is likewise very reasonable that still higher order effects remain small.

The notion of the W strongly interacting with itself is certainly not 
a new one. ${ }^{4,13}$ Among the proposals are $\mathrm{W}^{ \pm}$interacting strongly with ordinary hadrons, ${ }^{15}$ of $\mathrm{W}^{ \pm}$interacting strongly (i.e., nonrenormalizably) with photons, ${ }^{14}$ and of triplets or octets ${ }^{16}$ of $W^{\prime} \mathrm{s}$ with broken Yang-Mills coupling to each other. And while attractive from the point of view of S-matrix dynamics, the option of strongly coupled $\mathrm{W}$ is clearly anything from compelling. We can, however, anticipate the following general consequences:

1. There will be a large family of states of various spin and charge coupled to W. This argument is as good (or bad) as those advanced for the ordinary hadron system.

2. The W probably lies on a Regge trajectory. If it is exchangedegenerate, or approximately so, and if $J=0$ is physical, the spinless $W^{ \pm}$will be lighter than $\mathrm{J}=1 \mathrm{~W}^{ \pm}$, and one must take heed of the cautions of T. D. Lee ${ }^{31}$ regarding $\mathrm{W}$-searches via leptonic decay modes.

3. The slope of the W-trajectory is determined in order of magnitude by the lightest particle coupled strongly to the W-system. For example, if the W couples strongly to ordinary hadrons, the slope of the W-trajectory should be very large, of order $\left(\mathrm{m}_{\mathrm{P}} \mathrm{m}_{\mathrm{W}}\right)^{-1}$, as can be seen by comparing the Low equation ${ }^{32}$ for meson-W scattering with that of meson-nucleon scattering. The mean spacing in mass of $\mathrm{W}^{ \pm}$resonances in this case would be of order of meson masses.

4. To resolve the difficulties discussed under Hypothesis 4 and 5, one requires a strong damping of $\mathrm{J}=1 \mathrm{~W}^{+}-\mathrm{W}^{-}$interactions. From Cutkosky's work on vector-meson bootstraps, ${ }^{33}$ an attractive $\mathrm{W}^{+}-\mathrm{W}^{-}$force coming from a $J=1 W^{0}$ exchange might be strong enough to bootstrap the $W^{0}$. This again suggests the relevance of some kine of Yang-Mills multiplets of $J=1 W^{\prime} s$. The whole multiplet should again lie on Regge trajectories. 


\section{HIGH ENERGY LEPTON PROCESSES AND GARYONS}

If there exists $\mathrm{W}^{ \pm}$strongly coupled to a family of particles (which we shall call garyons), then one can crudely estimate cross-sections for high-energy lepton-lepton processes.

We here assume that the mass of this garyon family is characteristically of order $m_{w}$. The case of garyon = hadron has difficulties and will be discussed separately at the end of this section. At the other extreme, the cases in which weak interactions are linked with the electromagnetic interaction, whether by the nonrenormalizable quantum electrodynamics of the $\mathrm{W}$ as discussed by Lee and Yang, or by a symmetry between weak and electromagnetic processes, it may well be that the slope of the $\mathrm{W}$ trajectory is of order $\alpha$, i. e., $\mathrm{m}_{\mathrm{W}}^{*} / \mathrm{m}_{\mathrm{W}} \sim \sqrt{137} \sim 10$, and there is not only one mass scale. Therefore we do not expect the analysis of this section to necessarily apply to such cases.

The one-W-exchange (OWE) contribution to lepton-lepton scattering damps the high-energy "elastic" cross section, which eventually approaches a constant; for example

$$
\sigma_{\mathrm{OWE}}\left(\nu_{\mu}+\mathrm{e} \rightarrow \mu+\nu_{\mathrm{e}}\right) \underset{\mathrm{s} \rightarrow \infty}{\longrightarrow} \frac{2}{\pi} \mathrm{G}^{2} \mathrm{~m}_{\mathrm{W}}^{2}
$$

In addition to this, there is the contribution from garyon production as shown in Figure 3. If the WW cross-section is helicity independent, then as $s \rightarrow \infty$,

$$
\begin{gathered}
\frac{d \sigma}{d s^{\prime} d t^{\prime} d t_{2}}=\left(\frac{G m w^{2}}{2 \pi^{2}}\right)^{2} \frac{t_{1}}{\left(t_{1}+m_{w}^{2}\right)^{2}} \frac{t_{2}}{\left(t_{2}+m_{w}^{2}\right)^{2}} \\
\times \frac{1}{s^{\prime}} \log \frac{s}{s^{\prime}} \sigma_{w w}\left(s^{\prime}, t_{1}, t_{2}\right)
\end{gathered}
$$


Ignoring logarithmic factors, and taking the integrations over $t_{1}$ and $t_{2}$ to yield factors of order unity, we get as $s \rightarrow \infty$

$$
\sigma_{\ell \ell} \sim\left(\frac{\mathrm{Gm}_{\mathrm{w}}^{2}}{2 \pi^{2}}\right)^{2} \sigma_{\mathrm{ww}}\left(\mathrm{s}^{\dagger} \rightarrow \infty\right)
$$

Using the estimate given for the Froissart bound $\sigma_{\mathrm{ww}} \lesssim \pi / \mathrm{m}_{\mathrm{w}}{ }^{2}$ (assuming the lightest garyon state of charge two to be the two-W state) we get

$$
\sigma_{\ell \ell} \sim \frac{\mathrm{G}^{2} \mathrm{~m}_{\mathrm{w}}^{2}}{4 \pi^{3}}
$$

Thus for both the elastic and inelastic lepton-lepton cross-sections, the smooth linear rise of the cross section with s given by the Fermi theory is cut off at $\mathrm{s} \sim \mathrm{m}_{\mathrm{w}}^{2}$.

However, careful distinction must be made between lepton-lepton processes and those lepton-antilepton processes which can proceed via the single $\mathrm{W}^{ \pm}$, as in Figure 4. This can be estimated as follows: For $\mathrm{s} \gg \mathrm{m}_{\mathrm{W}}{ }^{2}$ but $\mathrm{s} \lesssim \mathrm{G}^{-1}$, the cross section for, say $\bar{\nu}_{\mathrm{e}}+\mathrm{e} \rightarrow$ garyons is bounded above by unitarity : $\sigma<$ const/s. Because this is the only contribution to the dispersion relation (4.8) of order $G$, we must have

$$
4 \sqrt{2} \mathrm{G}=\frac{1}{\pi} \int_{0}^{\infty} \frac{\mathrm{d} s^{\prime}}{s^{\prime}} \sigma_{\mathrm{TOT}}^{\left(\mathrm{s}^{\prime}\right)}
$$

Assuming that $\sigma \sim\left(\right.$ const) $\mathrm{s}^{-1}$ for $\mathrm{s}>\mathrm{m}_{\mathrm{w}}{ }^{2}$ gives us the rough upper bound

$$
\sigma^{\bar{\nu} \mathrm{e}}(\mathrm{s}) \lesssim \frac{4 \pi \mathrm{Gm}_{\mathrm{w}}{ }^{2}}{\mathrm{~s}}
$$

These contributions are schematically shown in Figure 5; they should 
certainly not be taken more seriously than to a factor of 10 .

If we try to return closer to reality and discuss lepton-hadron or hadron-hadron collisions, we may try using the parton model as a crude qualitative guide as to what to expect. The semileptonic lepton-hadron processes will be analogous to the lepton-lepton process, where no annihilation channel is present. Thus the observed linearly rising $\nu-\mathrm{N}$ total cross-section should, in this picture, but cut off at $\mathrm{s} z \mathrm{~m}_{\mathrm{w}}{ }^{2}$. The neutrino-production of garyons from hadrons should be within two orders of magnitude of the ordinary non-garyon production for $\mathrm{s} \gg \mathrm{m}_{\mathrm{w}}{ }^{2}$ :

$$
\frac{\sigma(1+\mathrm{n} \rightarrow 1+\text { garyons }+ \text { hadrons })}{\sigma(1+\mathrm{n} \rightarrow \text { all })} \sim \text { constant }
$$

However, for hadron-hadron (or photon-hadron) collisions, the anninilation channel is always open, and the cross-section should be more like the $\ell \bar{l}$ estimate, but probably suppressed considerably (1-2 orders of magnitude?), because only a small fraction of the available center-of-mass energy will on the average be in the parton-antiparton pair which annihilates into the single $\mathrm{W}$. Before leaving this subject, we must emphasize that in many cases, mixed electromagnetic and weak processes will result in larger garyon production cross-sections than stated herein. However, these considerations fall outside the purview of this paper.

We close this section by considering the possiblity that the $\mathrm{W}$ boson has strong pairwise interactions with ordinary hadrons. If this is the case, there are many constraints ${ }^{34,35}$ from experiment on its properties. We will examine those coming from inelastic neutrino scattering. We take the cross 
section for $\nu+\mathrm{N} \rightarrow \mu+$ anything (Figure 6) to be

$$
\frac{\mathrm{d} \sigma_{\nu \mathrm{N}}}{\mathrm{dt} \mathrm{ds}^{\prime}}=\sqrt{2} \frac{\mathrm{GM}_{\mathrm{W}}^{2}}{\pi^{2}} \frac{\mathrm{t}}{\left(\mathrm{t}+\mathrm{M}_{\mathrm{W}}^{2}\right)^{2}} \frac{\sigma_{\mathrm{WN}}(\mathrm{s})}{\mathrm{s}}
$$

and put it into the forward $\nu \mathrm{N}$ dispersion relation

$$
\mathrm{T}(\mathrm{s}, 0)=\frac{\mathrm{s}}{\pi} \int_{0}^{\infty} \mathrm{d} \mathrm{s}^{\prime}\left[\frac{\sigma_{\mathrm{TOT}}^{\nu N}\left(\mathrm{~s}^{\prime}\right)}{\mathrm{s}^{\prime}-\mathrm{s}}-\frac{\sigma_{\mathrm{TOT}}^{\bar{\nu}}\left(\mathrm{s}^{\prime}\right)}{\mathrm{s}^{\prime}+\mathrm{s}}\right]
$$

Neglecting logarithmic factors, we get at low energies (in order of magnitude only)

$$
\begin{aligned}
& \mathrm{T}(\mathrm{s}, 0) \sim \frac{\mathrm{GM}_{\mathrm{W}}^{2}}{\pi^{3}} \mathrm{~s} \int_{0}^{\infty} \frac{\mathrm{ds}^{\prime}}{\mathrm{s}^{\dagger}}\left[\sigma_{\mathrm{W}^{+} \mathrm{N}}\left(\mathrm{s}^{\prime}\right)-\sigma_{\mathrm{W}-\mathrm{N}^{-}}\left(\mathrm{s}^{\prime}\right)\right] \\
& +\frac{\mathrm{GM}_{\mathrm{W}}^{2}}{\pi^{3}} \mathrm{~s}^{2} \int_{0}^{\infty} \frac{\mathrm{ds^{ \prime }}}{\mathrm{s}^{2}}\left[\sigma_{\left.\mathrm{W}^{+} \mathrm{N}^{\left(\mathrm{s}^{\prime}\right)}+\sigma_{\mathrm{W}-\mathrm{N}}\left(\mathrm{s}^{\prime}\right)\right]}\right]
\end{aligned}
$$

This gives

$$
\mathrm{T} \sim \frac{\mathrm{Gs}}{\pi^{3}}\left\langle\sigma_{\mathrm{W}^{+} \mathrm{N}}-\sigma_{\mathrm{W}^{-} \mathrm{N}}\right\rangle_{\text {ave }} \quad \mathrm{M}_{\mathrm{W}}^{2}
$$

which will be much larger than experiment allows unless $\sigma+$ are identical. $^{36}$

To see this in detail, we note that the process in Figure 6 is much larger than conventional neutrino interactions. For $\mathrm{s}_{2} \mathrm{M}_{\mathrm{W}}{ }^{2}$,

$$
\frac{\sigma_{\nu \mathrm{N}} \rightarrow \text { garyons }}{\sigma_{\nu \mathrm{N} \rightarrow \text { hadrons }}} \sim \frac{\mathrm{GM}_{\mathrm{W}}{ }^{2}{ }^{\sigma} \mathrm{WN}}{\mathrm{G}^{2} \mathrm{M}_{\mathrm{W}}{ }^{2}} \sim \frac{\sigma_{\mathrm{WN}}}{\mathrm{G}}
$$


For a geometrical $\mathrm{W}-\mathrm{N}$ cross-section, this ratio is $\sim 10^{5 \pm 1}$ and can be shown 36,37 to be inconsistent with the low muon fluxed found in the deep mine experiments for $\mathrm{M}_{\mathrm{W}} \lesssim 10 \mathrm{GeV}$. But then the estimate of (6.11) for the low energy $\nu \mathrm{N}$ elastic scattering amplitude is greater than the conventional theory ${ }^{38}$ by $\sim 2$ orders of magnitude, unless $\sigma_{\mathrm{W}^{+} \mathrm{N}} \approx \sigma_{\mathrm{W}^{-} \mathrm{N}} \cdot$ Even if $\sigma_{\mathrm{W}_{\mathrm{p}}^{ \pm}}$differ only by electromagnetic effects, one may have difficulty with the existing experiments at low energies. Finally, even if the first term in $(6.10)$ can be neglected, the second rises rapidly with energy, giving

$$
\frac{\sigma_{\bar{\nu} \mathrm{N} \rightarrow \bar{\nu} \mathrm{N}}}{\sigma_{\bar{\nu} \mathrm{p} \rightarrow \mu^{+} \mathrm{n}}} \sim[\text { constant } 0(1)]\left[\mathrm{s} \sigma_{\mathrm{WN}}\right]^{2}
$$

independent of $\mathrm{m}_{\mathrm{W}^{*}}$. For the inelastic cross-sections this ratio is also probably similar. We conclude that either $\sigma_{\mathrm{WN}}$ is much less than geometrical or the hypothesis $\mathrm{W}=$ hadron, despite its very large mass, is either ruled out or can soon be decided by experiment. 


\section{ACKNOWLEDGMENTS}

One of us (JDB) acknowledges an important discussion long ago with M. Perl. In addition, he is grateful for a most stimulating and pleasant discussion with our Russian colleagues V. M. Shekhter, B. L. Ioffe, and L. B. Okun on the ideas of Pomeranchuk (reference 26). Those ideas and point of view to a considerable degree provide the stimulus for much of this work. In addition, we have benefitted from discussions with $\mathrm{K}$. Johnson, S. Glashow, N. Kroll, M. Einhorn, and many other colleagues at Harvard and SLAC. 


\section{REFERENCES AND FOOTNOTES}

1. T. D. Lee and C. N. Yang, Phys. Rev. Letters $\underline{4}, 307$ (1960).

2. B. L. Ioffe, L. B. Okun, and A. P. Rudik, Soviet Physics JETP 20 , 1281 (1965).

3. B. L. Ioffe and E. P Shabalin, Soviet J. Nucl. Phys. $\underline{6}, 603$ (1968).

4. R. E. Marshak, Riazuddin, C. P. Ryan, Theory of Weak Interactions in Particle Physics, Wiley-Interscience, New York (1969).

5. R. N. Mohapatra, J. S. Rao and R. E. Marshak, Phys. Rev. Letters 20 , 1081 (1968). Phys. Rev. 171,1502 (1968). P. Oleson, University of Rochester (unpublished).

6. M. Gell-Mann, M. L. Goldberger, N. M. Kroll and F. E. Low, Phys. Rev. $\underline{179}, 1518$ (1969).

7. A. R. Clark, T. Elioff, R. C. Field, H. J. Frisch, R. P. Johnson, L. T. Kerth and W. A. Wenze1, UCRL-20289 (1971), Phys. Rev. Letters 26, 1667 (1971).

8. Proceedings of the Fourth Annual Rochester Conference on High Energy Nuclear Physics, 1954.

9. A. O. Barut, Strong Interactions and High Energy Physics (London: Oliver and Boyd, 1969), pp. 81-104.

10. S. Mandelstam, Phys. Rev. 112,1344 (1958); 115, 1741, 1752 (1959).

11. J. Schwinger, Ann. Phys. 2, 407 (1957).

12. T. D. Lee and C. N. Yang, Phys. Rev. 108, 1611 (1957).

13. Gary Feinberg, Phys. Rev. 134, B1295 (1964).

14. T. D. Lee and C. N. Yang, Phys. Rev. 128, 885 (1962), and T. D. Lee, Phys. Rev. 128,899 (1962). 
15. T. Ericson and S. L. Glashow, Phys. Rev. 133B, 130 (1964). S. V. Pepper, C. Ryan, S. Okubo and R. E. Marshak, Phys. Rev. 137B, 1259 (1965). C. G. Callan, Jr., Phys. Rev. Letters $\underline{20}$, 809 (1968). R. N. Mohapatra, J. S. Rao and R. E. Marshak, Phys. Rev. 171, 1502 (1968).

16. C. Ryan, S. Okubo, and R. E. Marshak, Nuovo Cimento 34, 853 (1964), S. Okubo, Nuovo Cimento $\underline{54}$, 492 (1968), R. E. Marshak, Y. W. Yang, and J. S. Rao, Phys. Rev. D3, 1640 (1971), M. Gell-Mann, Proceedings of the 1969 Coral Cables Conference, Gordon and Breach, New York p-380.

17. G. Feinberg and F. Gursey, Phys. Rev. 128,378 (1962). We ignore here the additional $\mathrm{U}(2)$ symmetry of the right-handed leptons which do not participate in the weak coupling.

18. T. D. Lee, Nuovo Cimento $\underline{35}, 945$ (1965). T. D. Lee and C. S. Wu, Annual Review of Nuclear Science 15, 381 (1965).

19. Throughout this paper, we consider cross-sections only for initially polarized lepton beams (including e and $\mu$ ), and do not include any initial-state spin average.

20. R. B. Stothers, Phys. Rev. Letters $\underline{24}$, 538 (1970).

21. H. J. Steiner, Phys. Rev. Letters 24 , 746 (1970).

22. F. Reines and H. S. Gurr, Phys. Rev. Letters 24,1448 (1970).

23. C. H. Albright, Phys. Rev. D2, 1271 (1970).

24. M. Jacob and G. C. Wick, Ann. Phys. 7 , 404 (1959).

25. For a discussion of Lagrangian counterterms for nonrenormalizable field theories see N. N. Bogoliubov and D. V. Shirkov, Introduction to the Theory of Quantized Fields, New York, Interscience Publishers, Inc., 1959. 
26. I. Ya. Pomeranchuk, Soviet Journal of Nuclear Physics 11, 477 (1970), V. S. Berezinsky, Preprint N42, P. N. Lebedev Physical Institute, Moscow, USSR (1970).

27. Some support for the validity of the Pomeranchuk theorem and the existence of the Froissart bound in the case of neutrino scattering is given by R. Rajaraman, Phys. Rev. $\underline{178}$, 2211 (1969).

28. We are assuming here the additional Pomeranchuk theorem lore that $\frac{\operatorname{Re} \mathrm{D}(\mathrm{s}, 0)}{\mathrm{Im} \mathrm{D}(\mathrm{s}, 0)} \rightarrow 0$ faster than logarithmically as $\mathrm{s} \rightarrow \quad$. This assure the absence of an additional real term of the form const $\times s$ in Eq. (4.3) coming from the contour at infinity -- a fixed pole at $\mathrm{J}=1$.

29. M. Froissart, Phys. Rev. 123,1053 (1961).

30. For a review, see A. Martin, "Rigorous results from field theory and unitarity", Lectures given at the International School of Subnuclear Physics, Erice (1970), CERN Report TH. 1181.

31. T. D. Lee, Columbia University Preprint NYO-1932(2)-175.

32. F. E. Low, Phys. Rev. 97,1392 (1955).

33. R. Cutkosky, Phys. Rev. 131,1888 (1963).

34. J. B. Kogut, Phys. Rev. 186,1540 (1969).

35. C. Callan, reference 15 .

36. P. K. Kabir and A. N. Kamal, Muon Flux Deep Underground and the Question of Strong W-Interactions, Rutherford High Energy Laboratory Preprint.

37. J. D. Bjorken, S. Pakvasa, W. Simmons, and S. F. Tuan, Phys. Rev. 184 , No. 5, 1345 (1969). 
Table 1

Two particle lepton-lepton s-channel amplitudes in terms

of $\mathrm{A}, \mathrm{B}, \mathrm{C}$

Reactions

$$
\begin{gathered}
\nu_{\mathrm{e}} \mu \rightarrow \nu_{\mu} \\
\nu_{\mu} \mathrm{e} \rightarrow \mu \nu_{\mathrm{e}} \\
\nu_{\mathrm{e}^{\nu_{\mu}}} \rightarrow \nu_{\mathrm{e}} \nu_{\mu} \\
\mathrm{e}_{\mu}-\mathrm{e} \mu
\end{gathered}
$$$$
\nu_{\mathrm{e}^{\mu}}^{\mu \rightarrow \nu_{\mathrm{e}}^{\mu}}
$$$$
\mathrm{e} \nu_{\mu} \rightarrow \mathrm{e} \nu_{\mu}
$$$$
\nu_{\mathrm{e}} \mathrm{e} \rightarrow \mathrm{e} \nu_{\mathrm{e}}
$$$$
\nu_{\mu} \mu \rightarrow \mu \nu_{\mu}
$$

$$
\begin{aligned}
& \nu_{\mathrm{e}}^{\nu} \mathrm{e} \\
& \nu_{\mu}{ }_{\mu} \rightarrow \nu_{\mathrm{e}}{ }^{\nu} \mathrm{e} \\
& \mu^{\nu} \mu \\
& \mathrm{e}{ }_{\mu \mu} \rightarrow \mathrm{ee} \\
& \rightarrow \mu \mu
\end{aligned}
$$

Amplitude

$$
A(s, t)
$$

$\mathrm{B}(\mathrm{s}, \mathrm{t})$

$\mathrm{C}(\mathrm{s}, \mathrm{t})$

$B(s, t)-B(s, u)$ 


\section{FIGURE CAPTIONS}

1. Invariant amplitudes for weak lepton-lepton scattering

2. W-lepton rescattering effects in graphs for lepton-lepton scattering

3. Garyon production in lepton-lepton collisions

4. Garyon production via lepton-antilepton annihilation

5. Possible behavior of lepton-lepton and lepton-antilepton total cross-sections if garyons exist

6. Diagram for inelastic neutrino-nucleon scattering 

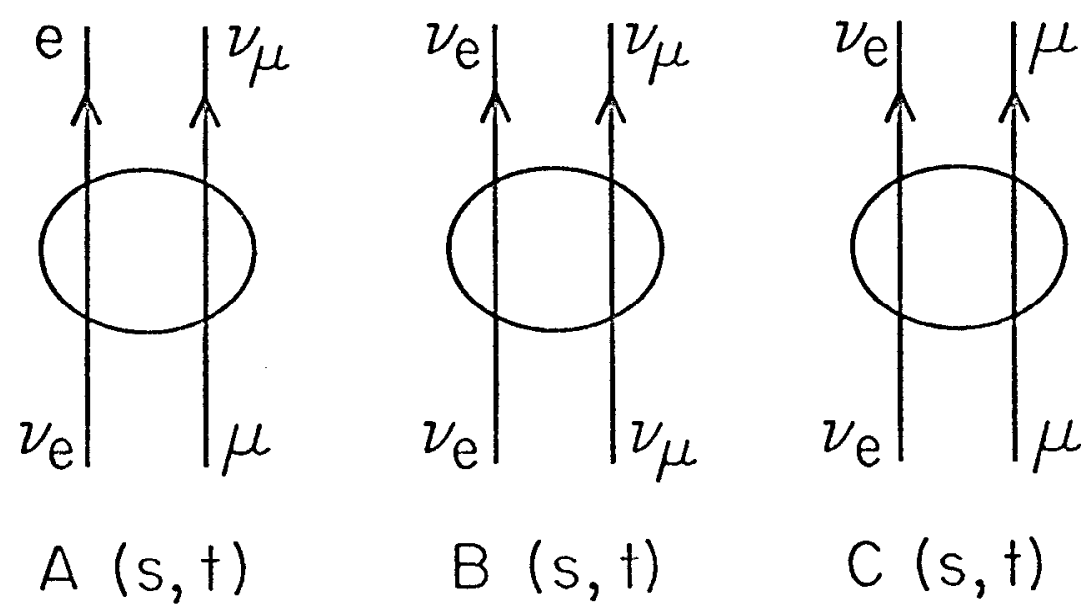

$B(s, t)$

$C(s, t)$

Fig. 1

1944A1 

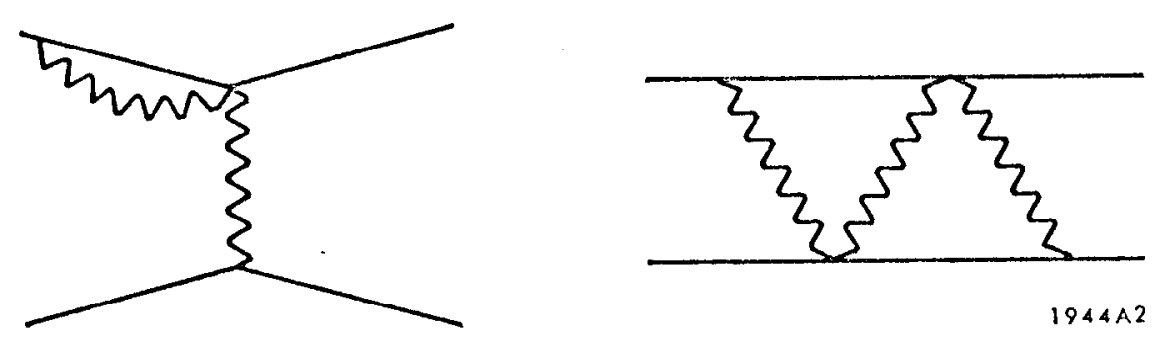

Fig. 2 


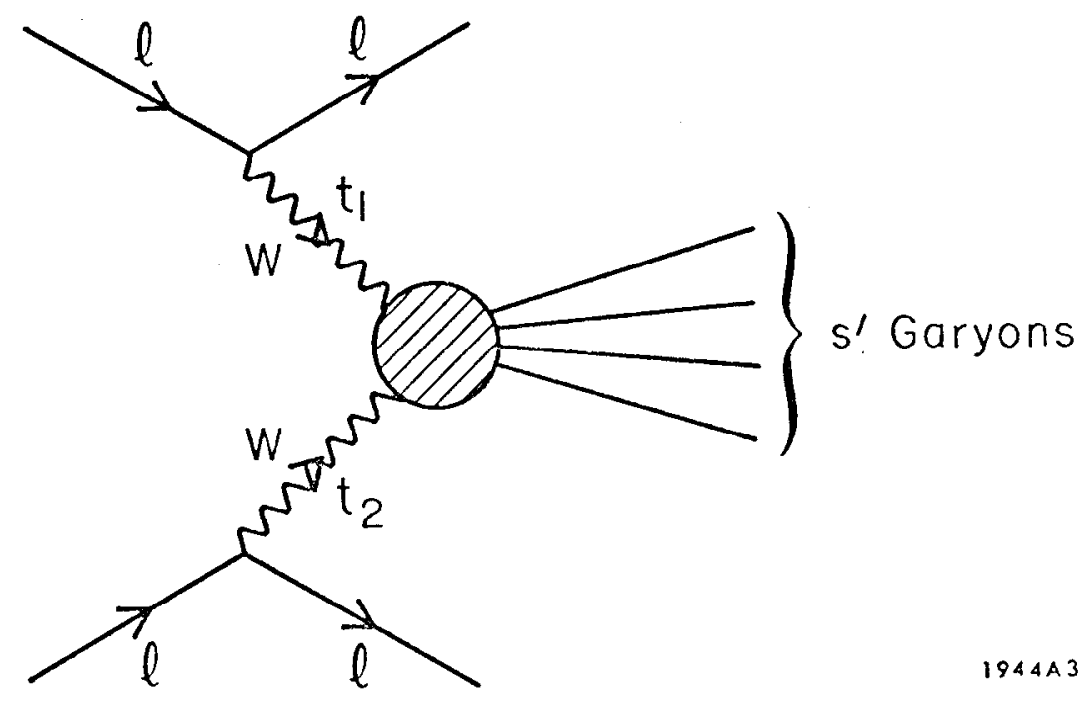

Fig. 3 


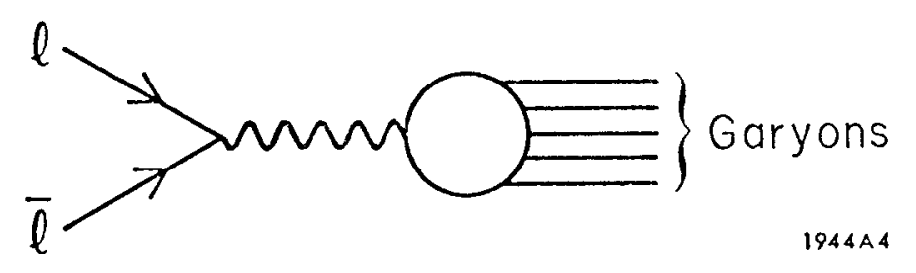

Fig. 4 


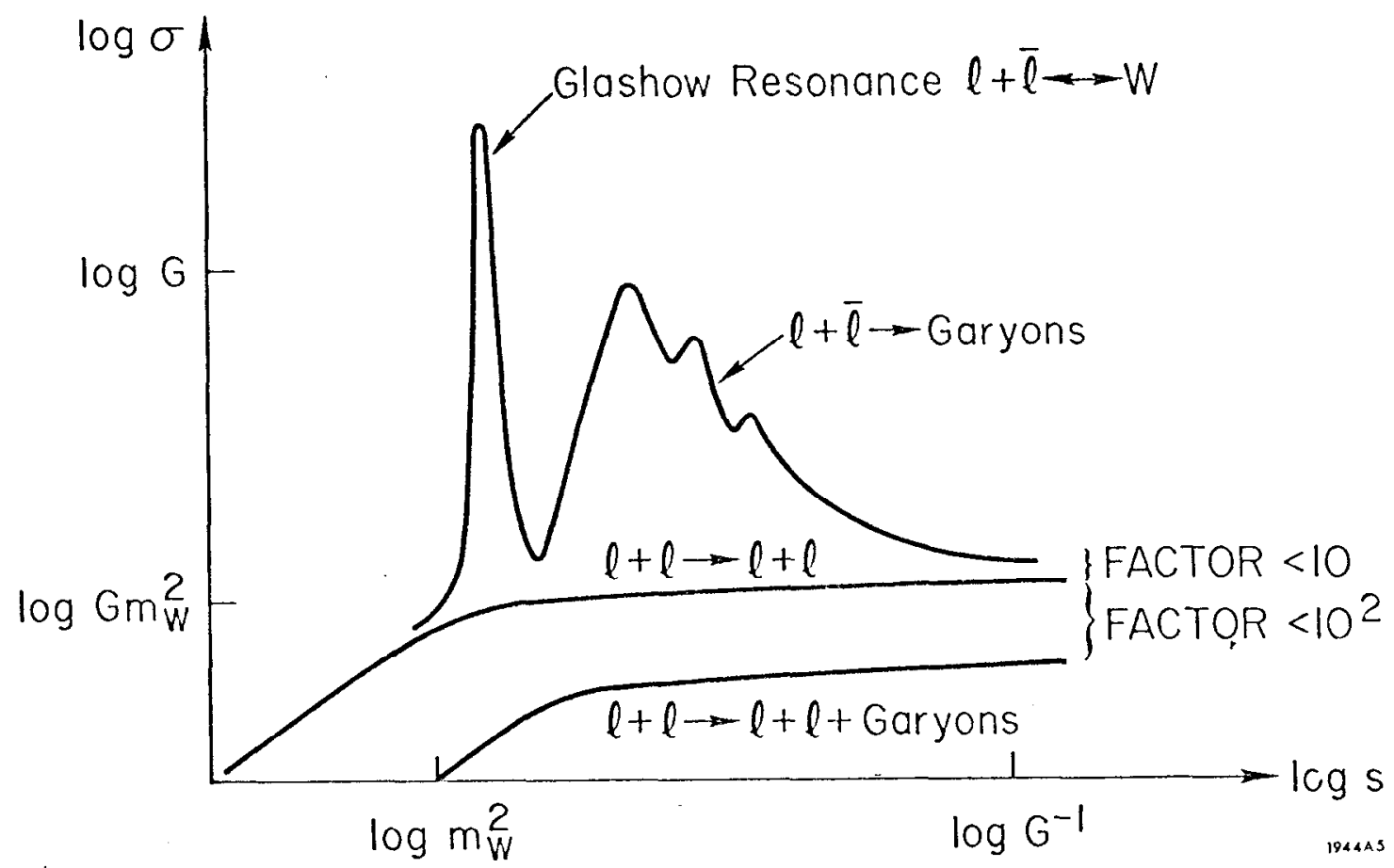

Fig. 5 


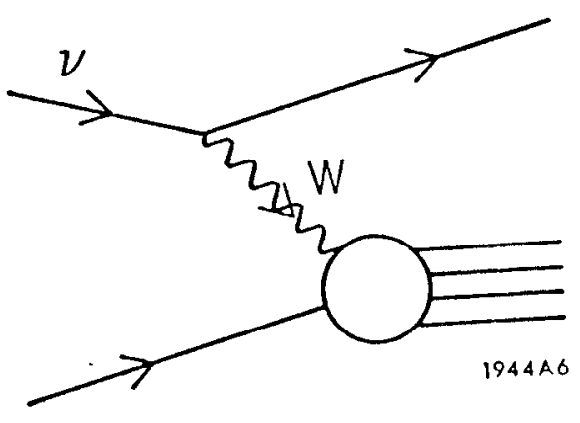

Fig. 6 